\title{
Ergonomics in Laparoscopy
}

\author{
${ }^{1}$ Meenakshi E Yeola (Pate), ${ }^{2}$ Dilip Gode, ${ }^{3}$ Akshay K Bora
}

\begin{abstract}
Ergonomics is the science of best suiting the worker to his job, or to make the setting and surroundings favorable for the laparoscopic surgeon. The term was formally defined in 1949 and has brought benefit and safety to many areas of human endeavor. ${ }^{1}$ The importance of ergonomics in the setting of laparoscopy cannot be overemphasized. Studies have shown that correct ergonomics can reduce suturing time. ${ }^{2}$ Pressurerelated chronic pain has been shown to be relieved by the use of ergonomically designed products. ${ }^{3}$ This article on ergonomics reviews the basic concepts and techniques, for example, triangulation, optimal coaxial alignment, drawbacks for the surgeon and the patient, and how to overcome these difficulties by recent advances in technology.
\end{abstract}

Keywords: Ergonomics, Hawthorne effect, Ideal position, Posture, Sectorization, Table height, Tactile sensation, Triangulation.

How to cite this article: Yeola (Pate) ME, Gode D, Bora AK. Ergonomics in Laparoscopy. Int J Recent Surg Med Sci 2017;3(2):102-108.

\section{Source of support: Nil}

\section{Conflict of interest: None}

\section{INTRODUCTION}

The use of minimal invasive procedures has increased tremendously over the last two decades. Compared with open surgery, the benefits of laparoscopic surgery include smaller incision, reduced postoperative morbidity and pain, shorter hospitalization, more rapid return to normal activities, and, in some instances, superior access. Laparoscopic surgery is different from open surgery in equipment, instrumentation, and psychomotor skills.

This article on ergonomics reviews the basic concepts and techniques, e.g., triangulation, optimal coaxial alignment, drawbacks for the surgeon and the patient, and how to overcome these difficulties by recent advances in technology.

\footnotetext{
${ }^{1}$ Professor, ${ }^{2}$ Resident, ${ }^{3}$ Professor and Vice Chancellor

${ }^{1-3}$ Department of Surgery, Jawaharlal Nehru Medical College Wardha, Maharashtra, India

Corresponding Author: Meenakshi E Yeola (Pate), Professor Department of Surgery, Jawaharlal Nehru Medical College Wardha, Maharashtra, India, Phone: +919822189896, e-mail: drmeenu7@rediff.com
}

\section{WHAT IS ERGONOMICS?}

- The term ergonomics is derived from the Greek words "ergon" meaning work and "nomos" meaning natural laws or arrangement.

- Ergonomics is "the scientific study of people at work, in terms of equipment design, workplace layout, the working environment, safety, productivity, and training." Ergonomics is based on anatomy, physiology, psychology, and engineering, combined in a systems approach.

- In simple words, it is the science of best suiting the worker to his job, or to make the setting and surroundings favorable for the laparoscopic surgeon. The term was formally defined in 1949 and has brought benefit and safety to many areas of human endeavor. ${ }^{1}$

\section{IMPORTANCE OF ERGONOMICS}

- The importance of ergonomics in the setting of laparoscopy cannot be overemphasized.

- Studies have shown that correct ergonomics can reduce suturing time. ${ }^{2}$

- Pressure-related chronic pain among surgeons has been shown to be relieved by the use of ergonomically designed products. ${ }^{3}$

\section{Hawthorne Effect}

- It has been a well-observed phenomenon that any individual performs a skill better and with more caution whenever he has the knowledge that he is under observation and assessment. This tends to skew the results toward more positive scores than would otherwise be obtained, if the person under study was unaware of the assessment being performed. This constitutes the "Hawthorne effect," which has been found applicable to most scientific assessments of human function and, hence, an integral knowledge of this aspect is essential for ergonomic purposes. ${ }^{4}$

- Laparoscopy, being a surgical skill performed by human dexterity and coordination, can definitely be assessed by ergonomic scales. Such assessments though need to be done secretively to avoid the bias introduced by the Hawthorne effect; there would arise multiple ethical and analytical problems in doing so. 


\section{Lack of Tactile Sensation}

- While learning the skills associated with open surgical procedures, as residents, we are trained to "see" with our hands as well as our eyes.

- We train our hands toward achieving this dual job in an attempt to reach the level of dexterity required to be competent. This constitutes the tactile feedback, which is conspicuously lacking when one transitions from performing open procedures to laparoscopic surgeries.

- The long graspers maneuvered through the trocars get substituted for the surgeon's hands, and this definitely reduces the efficiency and increases the time of the dissection. ${ }^{5}$

\section{Decreased Degree of Freedom of Movement}

- Open surgery has a high degree of freedom and surgeons work in line with visual axis. There is a three-dimensional (3D) direct vision and direct tactile feedback. While during laparoscopic surgery, there is a two-dimensional (2D) vision and loss of depth perception to some extent.

- Currently, there are only $4^{\circ}$ of freedom (rotation, up/down angulation, left/right angulation, in/out movement). According to Falk et $\mathrm{al}^{6}{ }^{6}$ an increase in the degree from 4 to 6 increases the dexterity by a factor of 1.5 .

- Additionally, there is the fulcrum effect with tremor enhancement. The major limitation is that view is not under control of the surgeon. ${ }^{7}$

\section{Decoupling of the Visual (Monitor) and Motor Axis}

- Visual orientation changes with the "loss of depth perception" due to indirect visual input and also the "loss of peripheral vision" or "Binocular effect" caused by the limited viewing spectrum offered.

- One of the most significant cognitive challenges for the general surgeon in his transformation into a laparoscopic surgeon is to overcome the spatial separation of the axis of vision and the axis of the physical aspect of the procedure.

- The surgeon does not get a chance to directly look at the instruments or his hands and also at the field of surgery at the same time. He has to learn to adapt to the difficulty of combining the two functions into the same-channeled approach in order to dexterously manipulate the tissues without direct contact.

- Studies have shown that working in separate coordinate systems decreases performance, leading to higher rates of error in the procedure. ${ }^{8}$

\section{Assumption of Relatively Static Posture}

- Great concentration and skill is required for performing the complex laparoscopic surgeries.
- Hence, it has been observed that the operating surgeon assumes a more static posture during laparoscopic procedures compared with the erstwhile open approach.

- These static postures have been demonstrated to be more disabling and harmful than dynamic postures are since muscles and tendons build up lactic acid and toxins when held for prolonged periods in same postures. $^{9-11}$

- Sensorial ergonomics (manipulations and visualization) improve precision, dexterity, and confidence, while physical ergonomics provide comfort for surgeon. Together, these two elements of ergonomics increase safety, have better outcome, and reduce the stress (Fig. 1). ${ }^{12}$

\section{More Clutter}

- Operative room (OR) crowding due to increase in the number of equipment, tubes, and cables in the $\mathrm{OR}^{13}$ creates physical hazards for traffic in the OR.

- The multitude of tubes and cables creates a "Spaghetti" of connections in the operating field that decreases the efficiency of instrument handling, positioning, and exchanges. ${ }^{14}$

- Using a ceiling-mounted boom system can make floor clutter free.

\section{Dark Room}

- Due to the OR lights being turned off during laparoscopic surgery, rest of the team must work in relative darkness.

- This increases the risk of choosing the wrong instruments and collision hazards.

\section{Drawbacks for the Surgeon}

- There have been multiple reports of carpal tunnel syndrome, eye strain, and cervical spondylosis among unsuspecting surgeons performing multiple

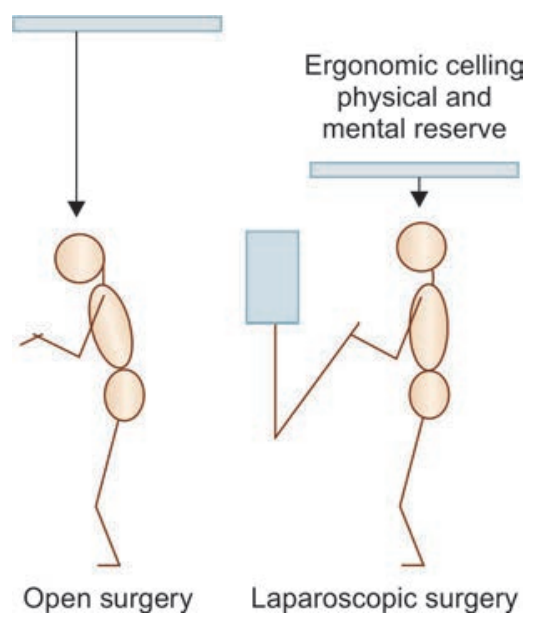

Fig. 1: The surgeon's mental and physical reserve during laparoscopic surgery is significantly reduced compared with open surgery 


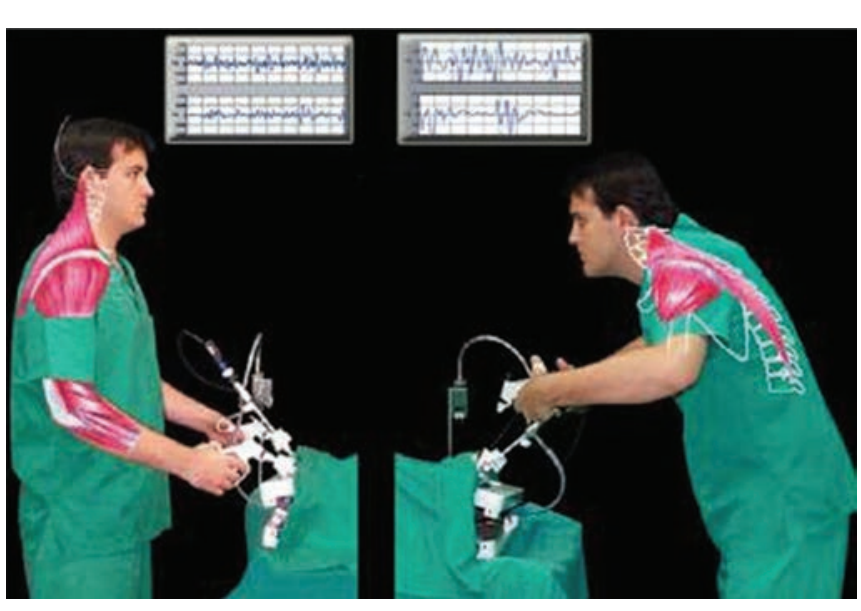

Fig. 2: Drawbacks for surgeon due to faulty posture and awkward thumb grip

laparoscopic procedures in high-volume centers. ${ }^{15}$ Reports of thenar neuropathy have arisen due to use of awkward thumb grips in case of laparoscopic pistol-grip instruments (Fig. 2). ${ }^{16}$

\section{Drawbacks for the Patient}

- Patients too have been found to be experiencing a lot of inconvenience with greater postoperative pain at port sites and due to other complications of the procedure.

- The mistakes leading to these poor outcomes seem to be completely avoidable with use of simple application of understanding of the physics and functioning of the whole event.

\section{Ergonomic Concepts in OR}

- The goal of proper posture is comfort, efficiency of movement, and minimization of the risk of musculoskeletal injuries to the operator.

- The surgeon's neck and back should be maintained in a comfortable and upright position facing forward.

- To achieve this ideal position, several factors are important.

\section{Ideal Position (Fig. 3)}

Visual angle to monitor

$15-40^{\circ}$ below line of sight

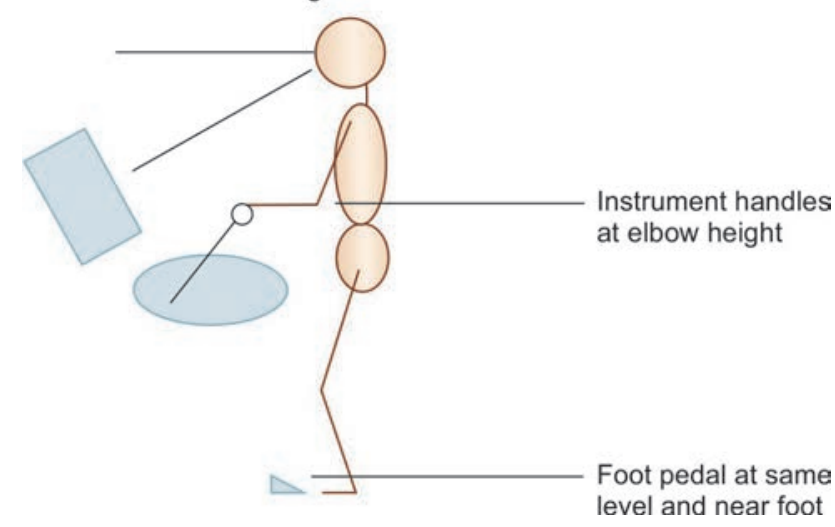

Fig. 3: Key elements of the ergonomic laparoscopic surgeon

\section{Factors affecting Ideal Position}

1. The height of the operating table

2. Position of the visual display (monitor)

3. Foot pedal location

4. Port placement

5. Related to instrumentation

6. Surgeon and team position

7. Technical advancements

\section{Operative Table Height}

- As in open surgery, the angle at the elbow joint should be between 90 and $120^{\circ}$ or, in simple terms, at or slightly below elbow level.

- The height of the table should be adjusted so as to achieve this goal.

- As laparoscopic instruments are longer and the table is also tilted much more than open surgery, this may require table to be lowered substantially. If this ideal position is not achieved, then the body unconsciously compensates for it by raising one side. This causes shoulder and neck strain, if the surgery is prolonged.

- If required, the surgeon needs to stand on an elevated platform if it is not possible to lower the table below a certain point. $^{17}$

\section{Monitor Position}

- Ergonomically, the best view for laparoscopy is with the monitor image at or within 15 to 40 (25 optimal) degrees below the horizontal plane of the eye. ${ }^{18,19}$ This leads to least neck strain according to the available studies.

- Standard liquid crystal display monitors placed on a low cart separate from the OR equipment may be used for best results. It is not advisable to have a "chin-up" arrangement on the part of the surgeon. ${ }^{19}$

- In operations where surgeons change their ports and positions, the second monitor is essential, e.g. total colectomy. Second monitor for assistants reduces strain on their neck.

\section{Foot Pedal Location}

- Foot pedals are commonly used during laparoscopic surgery to activate instruments, such as the cautery, ultrasonic shears, bipolar device, or other tissue welding/dividing instruments. Foot pedals, which are often poorly positioned, demand awkward and unnatural postures, and should be avoided in favor of hand controls when possible.

- Pedals should be placed near the foot and aligned in the same direction as the instruments, toward the target quadrant and the principal laparoscopic monitor. Such positioning will permit the surgeon to activate the pedal without twisting their body or leg. 
If the surgeon is standing on a lifting platform, the pedal must be placed at the same level off the ground.

- A pedal with a built-in foot rest is preferable so the surgeon does not have to hold their foot in the air or move it back and forth on the floor. If there are two pedals (for different devices), the surgeon must be careful not to confuse them in the darkness. ${ }^{20}$

\section{Port Placement}

- There is a need to understand some angles for better comprehension of port placement, i.e.,

- Manipulation

- Azimuth

- Elevation

\section{Manipulation Angles for Instrumentation}

- Manasnayakorn et $\mathrm{al}^{21}$ have studied in animal models and indicated that the best task efficiency and performance quality are obtained with an ideal manipulation angle between 45 and $60^{\circ}$ (Fig. 4).

- This can be achieved by correct placement of the ports. The $90^{\circ}$ manipulation angle had the greatest muscle workload by the deltoid and trapezius of the extracorporeal and intracorporeal limbs and the extracorporeal dominant arm extensor and flexor groups.

- Manipulation angle ranging from 45 to $75^{\circ}$ with equal azimuth angles is recommended. Manipulation angles below $45^{\circ}$ or above $75^{\circ}$ are accompanied by increased difficulty and degraded performance.

- Task efficiency was reported be better with equal azimuth angles than with unequal azimuth angles. Achieving equal azimuth angles may be difficult in many practical situations, but in principle, azimuth inequality should be avoided because it degrades task efficiency.

- There exists a direct correlation between the manipulation and the elevation angles.

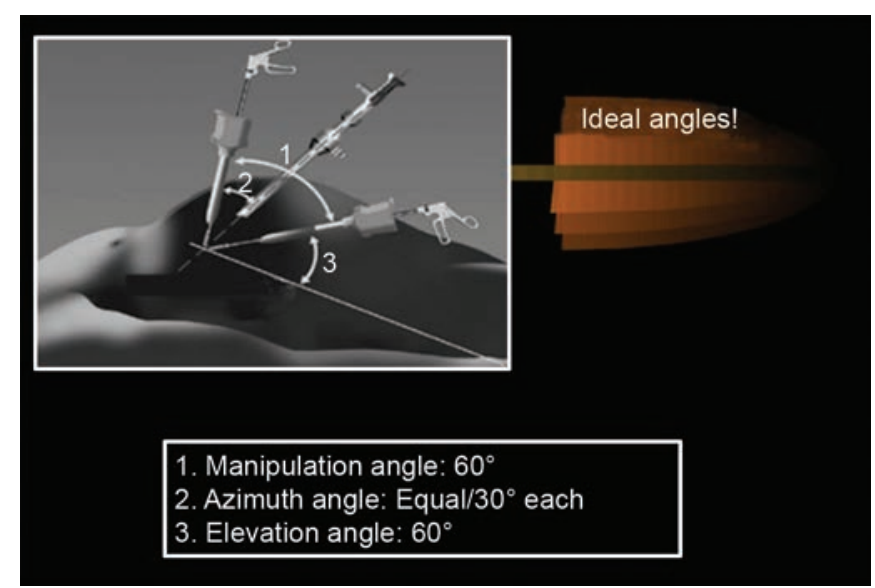

Fig. 4: Ideal angles
- With a manipulation angle of $60^{\circ}$, the corresponding optimal elevation angle that yields the shortest execution time and optimal quality performance is $60^{\circ}$.

- Wide manipulation angles necessitate wide elevation angles for optimal performance and task efficiency.

- When a $30^{\circ}$ manipulation angle is imposed by the anatomy or build of the patient, the elevation angle should be also $30^{\circ}$, as this combination carries the shortest execution time.

- The best ergonomic layout for endoscopic surgery consists of a manipulation angle ranging from 45 to $75^{\circ}$ with equal azimuth angles. ${ }^{22}$

\section{Triangulation}

- There is no uniform consensus about port placements for advanced laparoscopic procedures. The placement of ports is currently dictated by the surgeon's preference based on individual experience. To facilitate smooth instrument manipulation along with adequate visualization during laparoscopy, usually trocars are placed in triangular fashion. This is termed as triangulation.

- The target organ should be 15 to $20 \mathrm{~cm}$ from the center port used for placing the optical trocar. Generally, the two remaining trocars are placed in the same 15 to $20 \mathrm{~cm}$ arc at 5 to $7 \mathrm{~cm}$ on either side of the optical trocars.

- This allows the instruments to work at a 60 to $90^{\circ}$ angle ${ }^{20}$ with the target tissue, and avoids problems of long handle due to too far or too near placement of ports and the problem of abdominal wall interference. If necessary, two more retracting ports can be placed in the same arc, but more laterally so that instruments do not clash.

\section{Sectorization}

- When optical trocar is placed as one of the lateral port trocars, it is called as sectorization

- This is usually done during appendicectomy when a $10-\mathrm{mm}$ trocar is placed in the subumbilical region as optical trocar. Two other trocars are placed below these trocars laterally.

- Sectoring of instruments should be avoided by beginners, since it requires a greater degree of understanding and experience of the laparoscopic view and significantly different hand-eye coordination.

- Another factor that one should consider during trocar placement is that the instrument length is limited. If trocar is too far from the desired position, then one has to push the abdominal wall toward target organ to gain a few centimeters.

- This not only makes these movements less precise, but also causes strain on the finger and hand muscles. 
Similarly, if the angle between the target and instrument is too wide or obtuse, manipulation of curved instrument is very difficult. Most surgeons customise trocar position. If there is wrong placement of port, one has to push the abdominal wall and your hands start paining.

\section{Equipment-related Challenges}

- The importance of ergonomics in this field has been underscored by the US Food and Drug Administration reporting that probably half of the 1.3 million instrument-related injuries that occur in US hospitals each year could be due to poor instrument design. ${ }^{23}$

\section{Limited View}

- While performing minimal access surgery, the surgeon is typically viewing a 2D video image of the operating field on a television video screen placed at a certain distance of 4 to 8 feet away from the surgeon's eye. Even with the best-quality monitoring equipment, the quality and resolution details of the image are not comparable with direct visualization.

- Another limiting aspect which the laparoscopist has to contend with is the loss of peripheral vision, which was one of the cornerstones of his surgical skills in open procedures. He is no longer permitted to view anything besides the immediate field of the operation, and loses the luxury of efficient navigation in a larger surgical workspace.

- Use of angulated scopes can help achieve better view of anatomy in difficult situations. Normally, zero and a $30^{\circ}$ scopes are used, but more angulation can be used; however orientation is needed on the part of the surgeon due to limited view of field (Fig. 5).
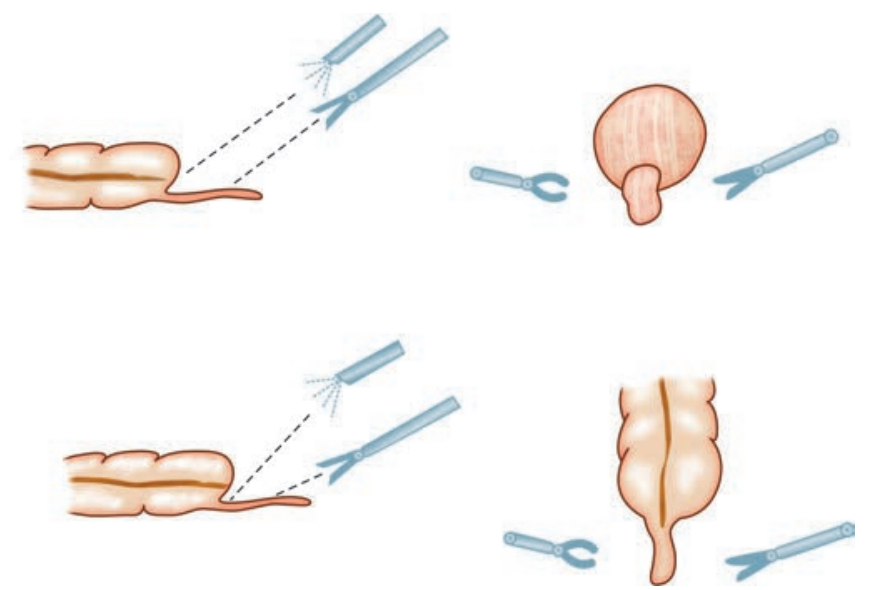

Fig. 5: View and orientation from a $0^{\circ}$ (above) and $30^{\circ}$ (below) scope

\section{Less Efficient Instruments}

- Laparoscopic instruments are constrained to work through small ports of 3 to $10 \mathrm{~mm}$ in size. This results in more complex internal mechanical linkages that decrease the efficient transmission of force from the surgeon's hand to the instrument tip.

- A typical disposable laparoscopic grasper transmits the force of the surgeon's hand from the handle to the tip with a ratio of only 1:3, in contrast to 3:1 ratio with a Hemostat. Hence, a laparoscopist has to work six times harder for similar results. ${ }^{24}$

- Moreover, these laparoscopic instruments are generally available in one standard size, and, hence, surgeons of all heights, builds, and hand sizes work with the same ones, and the efficacy suffers somewhere along the way. Customized instruments are prohibitively costly.

\section{Improperly Designed Shapes}

- Mattern and Waller ${ }^{25}$ have stated that improperly designed shapes of instruments cause strain on functional areas of the hand.

- They have designed a handle, i.e., based on ergonomic criteria. This multifunctional handle is shaped to fit only one hand and like a pistol handle, it rests continuously in the half-closed hand, similar to the "basic position" of the resting hand, between the ring and little fingers, with the thenar performing an encircling grip.

- The longitudinal axis of the instrument is an extension of the forearm's rotation axis. This allows pronation and supination to be transferred directly to the instrument effector.

\section{Limited Instrument Mobility}

- Laparoscopic technique requires the use of fixed position entry ports that limit the surgeon's ability to adjust instrument position and angle to the task at hand.

- Improperly placed ports can make an entire operation much more difficult to execute.

\section{Instrument Exchanges}

- These are laborious and distracting to the surgeon thus, placing a premium on minimizing exchanges and using multifunctional instruments. The latter, when poorly designed, can be even more difficult to use.

\section{Intracorporeal Suturing}

- Skill-related factors have a profound impact on the outcome. 
- These problems are a result of the necessity to suture in odd port positions in the absence of triangulation.

- Suturing at odd angles to the tissue, suturing in the retroperitoneum, and maintaining tension in continuous suturing while using less-efficient instruments.

\section{Surgeon and Team Position}

- A surgeon in two different positions can perform laparoscopic cholecystectomy. One is by standing on the left side of patients (preferred by Americans) and other is by standing between the legs (preferred by Europeans).

- Both the positions are convenient, but some find one more ergonomically better. It is usually surgeon's preference or habit of getting adjusted to the position. Though port placement is similar, there is a slight change in each position.

\section{Technical Advancements: Single Port Laparoscopy}

- Single port laparoscopy has changed the concept of triangulation used in conventional laparoscopy. With single port, the instruments often cross each other, making the procedure "counter-counterintuitive."

- To overcome these difficulties, steerable endoscopes, bent and articulating instrumentation, magnetic anchorage and guidance systems as well as flexible robotics have been developed.

\section{Role of Robotic Surgery}

- Robotic surgery is ergonomically advantageous as it has $7^{\circ}$ of freedom as compared with laparoscopic hand surgery. This helps one to access deeper areas, such as esophagus, pancreas, and retroperitoneum.

- It also allows placements of ports in shorter arc without instrument interference (Fig. 6).

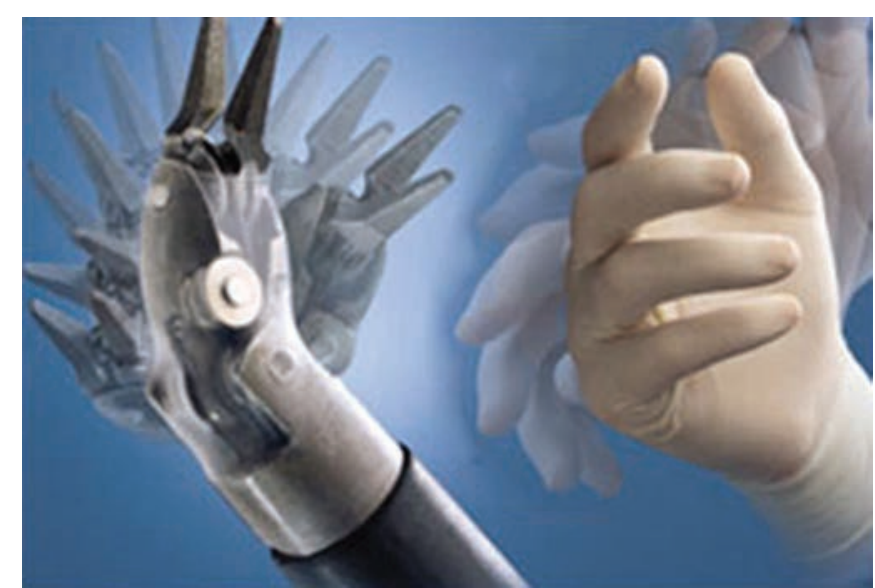

Fig. 6: Robotic surgery—degree of free movements
- In laparoscopic surgery, a significant benefit of 16:9 high-definition (HD) monitors would be that the images are of a natural, panoramic view. In humans, the horizontal field of view is wider than our vertical field of view.

- For surgeons, this wider, more natural view is less fatiguing during procedures.

- Additionally, during laparoscopic surgery, as surgeons are viewing full-screen endoscopic images, trocars and hand instruments that normally approach the surgical area laterally are visible easily with a 16:9 monitor rather than a $4: 3$ or 5:4 monitor.

\section{Three-dimensional Laparoscopy}

- The main advantage is accurate depth perception, which is very important for surgeries requiring suturing and other complex procedures.

- The 3D visualization of depth perception can shorten the learning curve for the surgeons.

\section{CONCLUSION}

- Laparoscopic surgery provides patients with less painful surgery, but is more demanding for the surgeon.

- The increased technological complexity and sometimes poorly adapted equipment have led to increased complaints of surgeon fatigue and discomfort during laparoscopic surgery.

- Better ergonomic integration and understanding ergonomics can not only make the life of the surgeon comfortable in the OR, but also reduce physical strains on the surgeon along with increasing productivity intraoperatively.

\section{CLINICAL SIGNIFICANCE}

Physical Constraints to Surgeons due to Inefficient Application of Ergonomics

- Neck pain and spondylosis has been observed to be a recurring complaint among surgeons in high-volume centers in the first decade after the advent of minimal access surgery. ${ }^{9}$

- The same height, at which the video monitor used to be set for surgeons of different heights, was found to be the underlying cause. ${ }^{9,10}$

- The other physical constraints reported are cervical spondylitis, shoulder pain due to abduction of shoulder (chicken wing scapula) during laparoscopy termed as "laparoscopic shoulder," backache, hand finger joint pain, tenosynovitis, burning eyes, stress exhaustion, and hand muscle injury. ${ }^{26}$ 


\section{Solution}

- The suggested position of arm is slightly abduction, retroversion, and rotation inward at shoulder level. The elbow should be bent at about 90 to $120^{\circ}$.

- The surgeon has to remember that moving about and loosening up his hands intermittently is essential to prevent the buildup of lactic acid and ward off fatigue.

- Problems related to depth perception, vision, and loss of peripheral visual fields can be reduced by using a 10 to $15 \times$ magnification on the optical system offered by the recording camera and the output to the display. This can make life easier while operating, especially, when dealing with minute and intricate internal anatomy.

- The lack of complete awareness among surgeons.

- Communication gap between the practitioners of laparoscopy and the designers of the instruments.

- Inadequate knowledge of the potential problems for the users of the instruments created by the designers.

- The contradictory expert advice, which reduces the credibility of ergonomics as a science.

\section{REFERENCES}

1. Kilbom A. Measurement and assessment of dynamic work. In: Wilson EC Jr, editor. Evaluation of human work: a practical ergonomics methodology. London: Taylor and Francis; 1990. p. 641-661.

2. Joice P, Hanna GB, Cuschieri A. Ergonomic evaluation of laparoscopic bowel suturing. Am J Surg 1998 Oct;176(4):373-378.

3. Van Veelen MA, Meiier DW. Ergnomics and design of laparoscopic instruments: results of a survey among laparoscopic surgeons. J Laparoendosc Adv Surg Tech A 1999 Dec;9(6):481-489.

4. Hanson DL. Evaluation of the Hawthorne effect on physical education research. Res Q 1967 Dec;38(4):723-724.

5. Patkin M, Isabel L. Ergonomics, engineering and surgery of endosurgical dissection. J Royal Coll Surg Edinb 1995 Apr;40(2):120-132.

6. Falk V, McLoughlin J, Guthart G, Salisbury JK, Walther T, Gummert J, Mohr FW. Dexterity enhancement in endoscopic surgery by a computer-controlled mechanical wrist. Minim Invasive Ther Allied Technol 1999;8(4):235-242.

7. Supe AN, Kulkarni GV, Supe PA. Ergonomics in laparoscopic surgery. J Minim Access Surg 2010 Apr-Jun;6(2):31-36.

8. Wang Y, MacKenzie CL. Effects of orientation disparity between haptic and graphic displays of objects in virtual environments. Human-computer interaction INTERACT ‘99. Edinburgh, Scotland: IOS Press; 1999.

9. Kant IJ, de Jong LC, van Rijssen-Moll M, Borm PJ. A survey of static and dynamic work postures of operating room staff. Int Arch Occup Environ Health 1992;63(6):423-428.
10. NguyenNT,HoHS,SmithWD, PhilippsC,LewisC,DeVera RM, Berguer R. An ergonomic evaluation of surgeons' axial skeletal and upper extremity movements during laparoscopic and open surgery. Am J Surg 2001 Dec;182(6):720-724.

11. Berguer R, Rab GT, Abu-Ghaida H, Alarcon A, Chung J. A comparison of surgeons' posture during laparoscopic and open surgical procedures. Surg Endosc 1997 Feb;11(2):139-142.

12. Stylopoulos N, Rattner D. Robotics and ergonomics. SCNA 2003 Dec;83(6):1321-1337.

13. Alarcon A, Berguer R. A comparison of operating room crowding between open and laparoscopic operations. Surg Endosc 1996 Sep;10(9):916-919.

14. Curtis P, Bournas N, Magos A. Simple equipment to facilitate operative laparoscopic surgery (or how to avoid a spaghetti junction). Br J Obstet Gynaecol 1995 Jun;102(6):495-497.

15. Hemal AK, Srinivas M, Charles AR. Ergonomic problems associated with laparoscopy. J Endourol 2001 Jun;15(5): 499-503.

16. Uchal M, Brogger J, Rukas R, Karlsen B, Bergamaschi R. Inline versus pistol-grip handles in a laparoscopic simulators. A randomized controlled crossover trial. Surg Endosc 2002 Dec;16(12):1771-1773.

17. De U. Ergonomics and laparoscopy. Indian J Surg 2005 May;67:164-166.

18. Menozzi M, von Buol A, Krueger H, Miege C. Direction of gaze and comfort: discovering the relation for the ergonomic optimization of visual tasks. Ophthalmic Physiol Opt 1994 Oct;14(4):393-399.

19. Hanna GB, Shimi SM, Cuschieri A. Task performance in endoscopic surgery is influenced by location of the image display. Ann Surg 1998 Apr;227(4):481-484.

20. Trejo A, Jung MC, Oleynikov D, Hallbeck MS. Effect of handle design and target location on the insertion and aim with a laparoscopic surgical tool. Appl Ergon 2007 Nov;38(6): 745-753.

21. Manasnayakorn S, Cuschieri A, Hanna GB. Ergonomic assessment of optimum operating table height for handassisted laparoscopic surgery. Surg Endosc 2009 Apr;23(4): 783-789.

22. Manasnayakorn S, Cuschieri A, Hanna GB. Ideal manipulation angle and instrument length in hand-assisted laparoscopic surgery. Surg Endosc 2008 Apr;22(4):924-929.

23. Rados C. FDA works to reduce preventable medical device injuries. FDA Consum 2003 Jul-Aug;37(4):29-33.

24. Forkey D, Smith W, Berguer R. A comparison of thumb and forearm muscle effort required for laparoscopic and open surgery using an ergonomic measurement station. 19th Annual International Conference of the IEEE Engineering in Medicine and Biology Society, Chicago, IL; 1997. p. 1705-1708.

25. Mattern U, Waller P. Instruments for minimally invasive surgery: principles of ergonomic handles. Surg Endosc 1999 Feb;13(2):174-182.

26. Berguer R, Forkey DL, Smith WD. The effect of laparoscopic instrument working angle on surgeons' upper extremity workload. Surg Endosc 2001 Sep;15(9):1027-1029. 